\title{
Quadratic pencil pole assignment by affine sums
}

\author{
S. Elhay* Yitshak M. Ram ${ }^{\dagger}$
}

(Received 8 August 2003; revised 28 December 2003)

\begin{abstract}
Differential equation models for damped vibrating systems are associated with quadratic matrix eigenvalue problems. The matrices in these systems are typically real and symmetric. The design and stabilisation of systems modelled by these equations require the determination of solutions to the inverse problem which are themselves real, symmetric and possibly with extra structure. In this paper we present a new method for pole assignment to a quadratic pencil by using affine sums. The method extends the work of Lancaster and Dai (1997) in which a similar problem for the generalized inverse eigenvalue problem is solved.
\end{abstract}

*Department of Computer Science, University of Adelaide, South Australia. mailto:elhay@cs.adelaide.edu.au

$\dagger$ Department of Mechanical Engineering, Louisiana State University, Baton Rouge, LA 70803-6413 USA. mailto:ram@me.lsu.edu

See http://anziamj.austms.org.au/V45/CTAC2003/Elha for this article, (c) Austral. Mathematical Soc. 2004. Published July 4, 2004. ISSN 1446-8735 


\section{Contents}

1 Introduction

C593

2 The method

C596

3 Examples and conclusions

C600

References

C602

\section{Introduction}

Applying separation of variables $\boldsymbol{v}(t)=\boldsymbol{z} e^{\lambda t}, \boldsymbol{z}$ a constant vector, to the second order matrix differential equation

$$
\boldsymbol{M} \boldsymbol{v}^{\prime \prime}+C \boldsymbol{v}^{\prime}+K \boldsymbol{v}=\mathbf{0},
$$

where a prime denotes differentiation with respect to time, leads to the problem of finding the eigenvalues and eigenvectors of the quadratic pencil

$$
P(\lambda)=\lambda^{2} \boldsymbol{M}+\lambda \boldsymbol{C}+\boldsymbol{K} .
$$

The scalar $\lambda_{i}$ is called an eigenvalue, and the corresponding vector $\boldsymbol{z}_{i} \neq \mathbf{0}$ is called an eigenvector of $P$, if they satisfy $P\left(\lambda_{i}\right) \boldsymbol{z}_{i}=\mathbf{0}$. Here, we will assume that $\boldsymbol{M}, \boldsymbol{C}$ and $\boldsymbol{K}$ are all in $R^{n \times n}$ (real, $n \times n$ matrices), symmetric and furthermore, that $\boldsymbol{M}$ is positive definite. Such pencils arise in the analysis of damped vibrating systems[3, e.g.].

In the direct quadratic eigenvalue problem we know the matrices $\boldsymbol{M}$, $\boldsymbol{C}$ and $\boldsymbol{K}$ and we seek the spectral properties of $P$ - the $2 n$ scalars $\lambda_{i}$ and their corresponding eigenvectors $\boldsymbol{z}_{i}$. The spectrum $\sigma(P)=\left\{\lambda_{i}\right\}_{i=1}^{2 n}$ of such a pencil forms a self-conjugate set, as do the set of eigenvectors, because the matrices here are real. 
For undamped systems $\boldsymbol{C}$ is a zero matrix and the problem reduces to the generalized eigenvalue problem in which the pencil is made linear $P(\mu)=$ $\boldsymbol{K}-\mu \boldsymbol{M}$ by the substitution $\mu=-\lambda^{2}$. Since $\boldsymbol{M}$ is positive definite, this pencil has $n$ real eigenvalues and $n$ linearly independent eigenvectors.

In the inverse problem we seek the matrices $\boldsymbol{M}, \boldsymbol{C}$ and $\boldsymbol{K}$, if they exist, which are such that the quadratic pencil they define has a prescribed spectrum. The fact that this spectrum contains complex elements adds a difficulty not present in the undamped case.

Inverse problems have become important in the theory of vibration and an excellent coverage of the field is to be found in [2]. Some affine sum methods for the inverse standard eigenvalue problem, where $\boldsymbol{M}$ vanishes and $\boldsymbol{C}$ is an identity are to be found in [1] and an affine sum method for the generalized inverse eigenvalue problem where $\boldsymbol{C}$ vanishes is dealt with in [4].

In this paper we present a new affine sum method for determining matrices $\boldsymbol{C}$ and $\boldsymbol{K}$, if such matrices exist, which together with the given matrix $\boldsymbol{M}$ define a quadratic pencil $P$ with a prescribed self-conjugate spectrum. More precisely, we address

\section{Problem 1 Given}

1. $M \in R^{n \times n}$ symmetric, positive definite,

2. $\left\{\boldsymbol{C}_{k}\right\}_{k=0}^{n},\left\{\boldsymbol{K}_{k}\right\}_{k=0}^{n}, \boldsymbol{C}_{k}, \boldsymbol{K}_{k} \in R^{n \times n}$, symmetric,

3. $S=\left\{\mu_{k}\right\}_{k=1}^{2 n}$, a self-conjugate set of scalars.

Define

$$
\boldsymbol{C}=\boldsymbol{C}_{0}+\sum_{k=1}^{n} \alpha_{k} \boldsymbol{C}_{k}, \quad \boldsymbol{K}=\boldsymbol{K}_{0}+\sum_{k=1}^{n} \beta_{k} \boldsymbol{K}_{k} .
$$

We seek real scalars $\left\{\alpha_{k}, \beta_{k}\right\}_{k=1}^{n}$, if they exist, which are such that the pencil (2) has spectrum $\sigma(P)=S$. 
To avoid a problem where the number of free parameters degenerates we will assume that the $n$ matrices $\left[\boldsymbol{C}_{k}, \boldsymbol{K}_{k}\right]$ are linearly independent in the space of $n \times 2 n$ matrices.

Denote the vector of target eigenvalues by $\boldsymbol{\mu}=\left(\mu_{1}, \mu_{2}, \ldots, \mu_{2 n}\right)^{T}$ and denote the coefficient vectors by $\boldsymbol{\alpha}=\left(\alpha_{1}, \alpha_{2}, \ldots, \alpha_{n}\right)^{T}$ and $\boldsymbol{\beta}=\left(\beta_{1}, \beta_{2}, \ldots, \beta_{n}\right)^{T}$. Also let $\boldsymbol{\lambda}(\boldsymbol{\alpha}, \boldsymbol{\beta})=\left(\lambda_{1}(\boldsymbol{\alpha}, \boldsymbol{\beta}), \lambda_{2}(\boldsymbol{\alpha}, \boldsymbol{\beta}), \ldots, \lambda_{2 n}(\boldsymbol{\alpha}, \boldsymbol{\beta})\right)^{T}$ denote the vector of eigenvalues of $P(\lambda)$ for particular $\boldsymbol{\alpha}, \boldsymbol{\beta}$ and, for each $i=1,2, \ldots, 2 n$, let $\boldsymbol{z}_{i}(\boldsymbol{\alpha}, \boldsymbol{\beta})$ be the eigenvector corresponding to the eigenvalue $\lambda_{i}(\boldsymbol{\alpha}, \boldsymbol{\beta})$.

In this paper we restrict ourselves to the case where $S$ does indeed define a solution to Problem 1 and we further assume that there is an open neighbourhood of $\boldsymbol{\alpha}, \boldsymbol{\beta}$ in which the conditions of Problem 1 are satisfied and $P(\lambda)$ has eigenvalues and eigenvectors which are analytic.

For a discussion of the conditions which need to be imposed on the set $S$ to ensure the existence of a solution $\boldsymbol{\alpha}, \boldsymbol{\beta}$ to Problem 1 see $[5,6,7]$. However, the constructions outlined there are restricted to rather special problems and importantly do not respect structure and so tridiagonality, sparsity and other structural properties are generally lost.

On the other hand, for affine solutions the family of matrices that make up the affine sums (3), aside from being symmetric, can also be chosen to have other important structural properties. Thus, for example, in the vibration control problem setting the family can be constructed from elements each of which represents a particular kind of control. There elements of the form $\left(\boldsymbol{e}_{i}-\right.$ $\left.\boldsymbol{e}_{j}\right)\left(\boldsymbol{e}_{i}-\boldsymbol{e}_{j}\right)^{T}$, where $\boldsymbol{e}_{k}$ is the $k$ th column of an identity matrix of appropriate dimension, represent passive controls. In addition, the nonlinearity of these problems frequently gives rise to a multiplicity of solutions, a feature which allows designers some choice in their designs.

In Section 2 we describe a Newton method for solving Problem 1 and in Section 3 we illustrate the method on some simple examples and mention come conclusions and further work. Empirical evidence suggests that the method has quadratic convergence. 


\section{The method}

In this section we develop a Newton method for the eigenvalues of the pencil $P$. The method is designed to find the values of affine sum coefficients $\boldsymbol{\alpha}$ and $\boldsymbol{\beta}$ which give $P$ the required eigenvalues.

We separate out real and imaginary parts of the complex eigenvalues and construct the method so that only real arithmetic is used throughout. Although the number of real and the number of complex eigenvalues can be different from one iteration to the next (typically early on in the iteration process) these numbers become fixed as the iteration approaches the values of $\boldsymbol{\alpha}$ and $\boldsymbol{\beta}$ to which the method converges. We therefore assume that the target eigenvalues and the eigenvalues for the current $\boldsymbol{\alpha}$ and $\boldsymbol{\beta}$ iterates have the same number of reals and complex pairs in what follows.

The target eigenvalues $\left\{\mu_{k}\right\}_{k=1}^{2 n}$ are ordered so that the first $r$

$$
\mu_{1} \leq \mu_{2} \leq \cdots \leq \mu_{r}
$$

are real and the remaining $2 n-r=2 c$ complex eigenvalues are arranged as conjugate pairs

$$
\begin{array}{cc}
\mu_{r+1}=\rho_{1}+i \eta_{1}, & \mu_{r+2}=\rho_{1}-i \eta_{1} \\
\mu_{r+3}=\rho_{2}+i \eta_{2}, & \mu_{r+4}=\rho_{2}-i \eta_{2}, \\
\vdots & \vdots \\
\mu_{2 n-1}=\rho_{c}+i \eta_{c}, & \mu_{2 n}=\rho_{c}-i \eta_{c} .
\end{array}
$$

Now consider the eigenvalues of the pencil for a particular set of coefficients $\boldsymbol{\alpha}$ and $\boldsymbol{\beta}$. We arrange these eigenvalues as we arranged the target eigenvalues: $\lambda_{1} \leq \lambda_{2} \leq \cdots \leq \lambda_{r}$ are real and the remaining $2 n-r=2 c$ complex eigenvalues and their eigenvectors are ordered thus

$$
\left.\begin{array}{ll}
\lambda_{r+2 j-1}=\phi_{j}+i \psi_{j} & \lambda_{r+2 j}=\phi_{j}-i \psi_{j} \\
\boldsymbol{z}_{r+2 j-1}=\boldsymbol{x}_{j}+i \boldsymbol{y}_{j} & \boldsymbol{z}_{r+2 j}=\boldsymbol{x}_{j}-i \boldsymbol{y}_{j}
\end{array}\right\} \quad j=1,2, \ldots, c .
$$


Finally, we partition the $2 n \times 2 n$ real Jacobian of $\boldsymbol{f}$ as

$$
\boldsymbol{J}=\left(\begin{array}{ll}
\boldsymbol{J}_{1} & \boldsymbol{J}_{2} \\
\boldsymbol{J}_{3} & \boldsymbol{J}_{4} \\
\boldsymbol{J}_{5} & \boldsymbol{J}_{6}
\end{array}\right) \quad \begin{gathered}
r \\
n
\end{gathered}
$$

where the real eigenvalue blocks are defined by $\left[\boldsymbol{J}_{1}\right]_{i j}=\partial \lambda_{i} / \partial \alpha_{j},\left[\boldsymbol{J}_{2}\right]_{i j}=$ $\partial \lambda_{i} / \partial \beta_{j}$, for all $i=1,2, \ldots, r$ and $j=1,2, \ldots, n$, and the blocks for the complex eigenvalues are defined by $\left[\boldsymbol{J}_{3}\right]_{i j}=\partial \phi_{i} / \partial \alpha_{j},\left[\boldsymbol{J}_{4}\right]_{i j}=\partial \phi_{i} / \partial \beta_{j}$, $\left[\boldsymbol{J}_{5}\right]_{i j}=\partial \psi_{i} / \partial \alpha_{j},\left[\boldsymbol{J}_{6}\right]_{i j}=\partial \psi_{i} / \partial \beta_{j}$, for all $i=1,2, \ldots, c, j=1,2, \ldots, n$.

We apply Newton's method to the $2 n$ dimension real valued function

$$
\boldsymbol{f}(\boldsymbol{\alpha}, \boldsymbol{\beta})=\left(\boldsymbol{v}_{\lambda}^{T}, \boldsymbol{v}_{\phi}^{T}, \boldsymbol{v}_{\psi}^{T}\right)^{T}
$$

which is defined in terms of the three real vectors $\boldsymbol{v}_{\lambda} \in R^{r}$ and $\boldsymbol{v}_{\phi}, \boldsymbol{v}_{\psi} \in R^{c}$ where, $\left[\boldsymbol{v}_{\lambda}\right]_{j}=\left(\lambda_{j}(\boldsymbol{\alpha}, \boldsymbol{\beta})-\mu_{j}\right), j=1,2, \ldots, r,\left[\boldsymbol{v}_{\phi}\right]_{j}=\left(\phi_{j}(\boldsymbol{\alpha}, \boldsymbol{\beta})-\rho_{j}\right)$, $j=1,2, \ldots, c$ and $\left[\boldsymbol{v}_{\psi}\right]_{j}=\left(\psi_{j}(\boldsymbol{\alpha}, \boldsymbol{\beta})-\eta_{j}\right), j=1,2, \ldots, c$, to find the real $\boldsymbol{\alpha}, \boldsymbol{\beta}$ which zero it.

Leaving aside the dependence on $\boldsymbol{\alpha}, \boldsymbol{\beta}$ where there is no ambiguity, we have for the eigenpair $\lambda_{i}, \boldsymbol{z}_{i}$,

$$
\boldsymbol{z}_{i}^{T}\left(\lambda_{i}^{2} \boldsymbol{M}+\lambda_{i} \boldsymbol{C}+\boldsymbol{K}\right) \boldsymbol{z}_{i}=0 .
$$

Note that here, as elsewhere, the quantity $\boldsymbol{z}_{i}^{T}$ denotes a true transpose and not a conjugate transpose. Denoting a derivative with respect to either $\alpha_{j}$ or $\beta_{j}$ by a dot, we differentiate (7) to get

$$
2 \dot{\boldsymbol{z}}_{i}^{T}\left(\lambda_{i}^{2} \boldsymbol{M}+\lambda_{i} \boldsymbol{C}+\boldsymbol{K}\right) \boldsymbol{z}_{i}+\boldsymbol{z}_{i}^{T}\left(2 \lambda_{i} \dot{\lambda}_{i} \boldsymbol{M}+\dot{\lambda}_{i} \boldsymbol{C}+\lambda_{i} \dot{\boldsymbol{C}}+\dot{\boldsymbol{K}}\right) \boldsymbol{z}_{i}=0 .
$$

This reduces, by (7), to

$$
\boldsymbol{z}_{i}^{T}\left(\dot{\lambda}_{i}\left(2 \lambda_{i} \boldsymbol{M}+\boldsymbol{C}\right)+\lambda_{i} \dot{\boldsymbol{C}}+\dot{\boldsymbol{K}}\right) \boldsymbol{z}_{i}=0
$$


We now get expressions for the derivatives of the real and imaginary parts of the eigenvalues from relation (9) in order to calculate the Jacobian matrix.

We deal first with the eigenvalues which are pure real. Provided the denominators do not vanish we isolate $\dot{\lambda}$ to get

$$
\dot{\lambda}_{i}=-\frac{\boldsymbol{z}_{i}^{T}\left(\lambda_{i} \dot{\boldsymbol{C}}+\dot{\boldsymbol{K}}\right) \boldsymbol{z}_{i}}{\boldsymbol{z}_{i}^{T}(2 \lambda \boldsymbol{M}+\boldsymbol{C}) \boldsymbol{z}_{i}} .
$$

Now note that for $j=1,2, \ldots, n$

$$
\frac{\partial \boldsymbol{C}}{\partial \alpha_{j}}=\boldsymbol{C}_{j}, \quad \frac{\partial \boldsymbol{C}}{\partial \beta_{j}}=\frac{\partial \boldsymbol{K}}{\partial \alpha_{j}}=\boldsymbol{O}, \quad \frac{\partial \boldsymbol{K}}{\partial \beta_{j}}=\boldsymbol{K}_{j},
$$

and so for $1 \leq i \leq r$

$$
\frac{\partial \lambda_{i}}{\partial \alpha_{j}}=-\frac{\lambda_{i} \boldsymbol{z}_{i}^{T} \boldsymbol{C}_{j} \boldsymbol{z}_{i}}{\boldsymbol{z}_{i}^{T}\left(2 \lambda_{i} \boldsymbol{M}+\boldsymbol{C}\right) \boldsymbol{z}_{i}}, \quad \frac{\partial \lambda_{i}}{\partial \beta_{j}}=-\frac{\boldsymbol{z}_{i}^{T} \boldsymbol{K}_{j} \boldsymbol{z}_{i}}{\boldsymbol{z}_{i}^{T}\left(2 \lambda_{i} \boldsymbol{M}+\boldsymbol{C}\right) \boldsymbol{z}_{i}} .
$$

This defines the $r \times 2 n$ first block row of the Jacobian matrix.

Writing the real and imaginary parts of (9) explicitly gives, (omitting subscripts in the interests of clarity)

$$
\begin{aligned}
& (\boldsymbol{x}+i \boldsymbol{y})^{T}\{[\dot{\phi}(2 \phi \boldsymbol{M}+\boldsymbol{C})-2 \dot{\psi} \psi \boldsymbol{M}+\psi \dot{\boldsymbol{C}}+\dot{\boldsymbol{K}}] \\
& \quad+i[\dot{\psi}(2 \phi \boldsymbol{M}+\boldsymbol{C})+2 \dot{\phi} \psi \boldsymbol{M}+\phi \dot{\boldsymbol{C}}]\}(\boldsymbol{x}+i \boldsymbol{y})=0 .
\end{aligned}
$$

We equate the real and imaginary parts of (13) to zero to find, after some manipulations,

$$
\dot{\phi}=\frac{u w+v t}{t^{2}+u^{2}}, \quad \dot{\psi}=\frac{t w-u v}{t^{2}+u^{2}},
$$

where

$$
\begin{aligned}
& t=(\boldsymbol{x}+\boldsymbol{y})^{T}(2 \phi \boldsymbol{M}+\boldsymbol{C})(\boldsymbol{x}-\boldsymbol{y})-4 \psi \boldsymbol{x}^{T} \boldsymbol{M} \boldsymbol{y}, \\
& u=2 \psi(\boldsymbol{x}+\boldsymbol{y})^{T} \boldsymbol{M}(\boldsymbol{x}-\boldsymbol{y})+2 \boldsymbol{x}^{T}(2 \phi \boldsymbol{M}+\boldsymbol{C}) \boldsymbol{y}, \\
& v=-(\boldsymbol{x}+\boldsymbol{y})^{T}(\phi \dot{\boldsymbol{C}}+\dot{\boldsymbol{K}})(\boldsymbol{x}-\boldsymbol{y})+2 \psi \boldsymbol{x}^{T} \dot{\boldsymbol{C} y}, \\
& w=-\psi(\boldsymbol{x}+\boldsymbol{y})^{T} \dot{\boldsymbol{C}}(\boldsymbol{x}-\boldsymbol{y})-2 \boldsymbol{x}^{T}(\phi \dot{\boldsymbol{C}}+\dot{\boldsymbol{K}}) \boldsymbol{y} .
\end{aligned}
$$


The numbers $t$ and $u$ are independent of $\dot{\boldsymbol{C}}$ and $\dot{\boldsymbol{K}}$ and so they remain the same whether the differentiation in (9) is with respect to $\alpha_{j}$ or $\beta_{j}$. But the numbers $v$ and $w$, when we differentiate with respect to $\alpha_{j}$ reduce, by virtue of (11), to $v=-(\boldsymbol{x}+\boldsymbol{y})^{T} \phi \dot{\boldsymbol{C}}(\boldsymbol{x}-\boldsymbol{y})+2 \psi \boldsymbol{x}^{T} \dot{\boldsymbol{C}} \boldsymbol{y}$ and $w=-(\boldsymbol{x}+\boldsymbol{y})^{T} \dot{\boldsymbol{C}}(\boldsymbol{x}-$ $\boldsymbol{y})-2 \boldsymbol{x}^{T} \phi \dot{\boldsymbol{C}} \boldsymbol{y}$ and these are used in (14) to compute the elements of the block components $\boldsymbol{J}_{3}$ and $\boldsymbol{J}_{5}$. By similar reasoning $u$ and $v$, for the case where we differentiate with respect to $\beta_{j}$, become $v=-(\boldsymbol{x}+\boldsymbol{y})^{T} \dot{\boldsymbol{K}}(\boldsymbol{x}-\boldsymbol{y})$ and $w=-2 \boldsymbol{x}^{T} \dot{\boldsymbol{K}} \boldsymbol{y}$, and these are used in (14) to compute the elements of the block components $\boldsymbol{J}_{4}$ and $\boldsymbol{J}_{6}$.

This completes the determination of $\boldsymbol{J}$ and we immediately write the step of the Newton method which computes the estimates $\boldsymbol{\alpha}^{(k+1)}$ and $\boldsymbol{\beta}^{(k+1)}$ from $\boldsymbol{\alpha}^{(k)}$ and $\boldsymbol{\beta}^{(k)}$ as

$$
\boldsymbol{J}\left(\boldsymbol{\alpha}^{(k)}, \boldsymbol{\beta}^{(k)}\right)\left(\left(\begin{array}{c}
\boldsymbol{\alpha}^{(k+1)} \\
\boldsymbol{\beta}^{(k+1)}
\end{array}\right)-\left(\begin{array}{c}
\boldsymbol{\alpha}^{(k)} \\
\boldsymbol{\beta}^{(k)}
\end{array}\right)\right)=-\boldsymbol{f}\left(\boldsymbol{\alpha}^{(k)}, \boldsymbol{\beta}^{(k)}\right) .
$$

The $k$ th step of the algorithm now operates as follows: using the current values $\boldsymbol{\alpha}^{(k)}$ and $\boldsymbol{\beta}^{(k)}$,

1. compute the eigendecomposition of $P\left(\lambda\left(\boldsymbol{\alpha}^{(k)}, \boldsymbol{\beta}^{(k)}\right)\right)$,

2. compute the Jacobian $\boldsymbol{J}\left(\boldsymbol{\alpha}^{(k)}, \boldsymbol{\beta}^{(k)}\right)$ using (12) and (14),

3. compute the right hand side function $\boldsymbol{f}\left(\boldsymbol{\alpha}^{(k)}, \boldsymbol{\beta}^{(k)}\right)$ of (6),

4. solve the linear system (15).

Stop when $\delta^{(k+1)} \stackrel{\text { def }}{=}\left\|\left(\begin{array}{c}\boldsymbol{\alpha}^{(k+1)} \\ \boldsymbol{\beta}^{(k+1)}\end{array}\right)-\left(\begin{array}{c}\boldsymbol{\alpha}^{(k)} \\ \boldsymbol{\beta}^{(k)}\end{array}\right)\right\|_{2}$ is sufficiently small. 


\section{$3 \quad$ Examples and conclusions}

In this section we present small, simple examples to illustrate the use of the method and to show the behaviour, typical in the experience of the authors, which supports the conjecture that the method has second order of convergence. All calculations were performed in IEEE standard double precision arithmetic (machine $\epsilon \approx 2 \times 10^{-16}$ ).

Example 1 In this example we have $n=5, \boldsymbol{M}=\boldsymbol{I}$,

$$
\boldsymbol{C}_{0}=\left(\begin{array}{rrrrr}
10 & -10 & 0 & 0 & 0 \\
-10 & 18 & -8 & 0 & 0 \\
0 & -8 & 12 & -4 & 0 \\
0 & 0 & -4 & 12 & -8 \\
0 & 0 & 0 & -8 & 11
\end{array}\right), \quad \boldsymbol{K}_{0}=\left(\begin{array}{rrrrr}
10 & -10 & 0 & 0 & 0 \\
-10 & 18 & -8 & 0 & 0 \\
0 & -8 & 12 & -4 & 0 \\
0 & 0 & -4 & 12 & -8 \\
0 & 0 & 0 & -8 & 11
\end{array}\right)
$$

and the affine families have $\boldsymbol{K}_{i}=\boldsymbol{C}_{i}=\left(\boldsymbol{e}_{i}-\boldsymbol{e}_{i+1}\right)\left(\boldsymbol{e}_{i}-\boldsymbol{e}_{i+1}\right)^{T}, i=$ $1,2, \ldots, n-1$ and $\boldsymbol{K}_{n}=\boldsymbol{C}_{n}=\boldsymbol{e}_{1} \boldsymbol{e}_{1}^{T}+\boldsymbol{e}_{n} \boldsymbol{e}_{n}^{T}$. For the case

$$
\boldsymbol{\alpha}=-\boldsymbol{\beta}=\left(\begin{array}{lllll}
-1 & 1 & -1 & 1 & -1
\end{array}\right),
$$

the pencil $P(\lambda)$ has the real eigenvalues $-26.07397,-18.47433,-8.91370$, $-2.48789,-1.11603,-0.36370$ and the complex eigenvalues $-1.69234 \pm$ $2.43496 i,-0.09285 \pm 0.72845 i$. Using the starting values

$$
\begin{aligned}
& \boldsymbol{\alpha}^{(0)^{T}}=(-1.2,1.4,-1.6,1.8,-2.0), \\
& \boldsymbol{\beta}^{(0)^{T}}=(1.2,-1.4,1.6,-1.8,2.0),
\end{aligned}
$$

the method finds the $\boldsymbol{\alpha}, \boldsymbol{\beta}$ shown in (16) correct to about twelve decimals in nine iterations (see Table 1). The rate at which the error reduces is consistent with second order convergence. 
TABLE 1: Successive correction term norms $\delta^{(k+1)}$ and function value norms for Example 1. Exponents are shown in parentheses.

\begin{tabular}{|cc||c|}
\hline$k$ & $\delta^{(k+1)}$ & $\boldsymbol{f}\left(\boldsymbol{\alpha}^{(k)}, \boldsymbol{\beta}^{(k)}\right) \|_{2}$ \\
\hline 0 & $5.33(+00)$ & $1.53(+00)$ \\
1 & $2.93(+00)$ & $2.62(-01)$ \\
2 & $1.12(+00)$ & $6.99(-02)$ \\
3 & $2.93(-01)$ & $1.09(-02)$ \\
4 & $6.83(-02)$ & $1.51(-03)$ \\
5 & $1.47(-02)$ & $2.07(-04)$ \\
6 & $7.91(-04)$ & $9.97(-06)$ \\
7 & $2.27(-06)$ & $2.85(-08)$ \\
8 & $1.91(-11)$ & $2.49(-13)$ \\
9 & $3.96(-13)$ & $3.89(-14)$ \\
\hline
\end{tabular}

Example 2 Using the same $\boldsymbol{M}, \boldsymbol{C}_{0}, \boldsymbol{K}_{0}$, affine family and $\boldsymbol{\alpha}, \boldsymbol{\beta}$ as in Example 1 but with the alternate starting values

$$
\begin{aligned}
& \boldsymbol{\alpha}^{(0)^{T}}=(-0.54551,1.29812,-0.83552,1.23910,-0.70141), \\
& \boldsymbol{\beta}^{(0)^{T}}=(1.08072,-0.58526,1.47806,-0.70223,1.01437),
\end{aligned}
$$

the method finds a second solution

$$
\begin{aligned}
& \boldsymbol{\alpha}^{(8)^{T}}=(-0.87850,0.89416,-0.99899,0.99300,-1.00968), \\
& \boldsymbol{\beta}^{(8)^{T}}=(1.11642,-1.08588,1.05144,-1.16071,1.01043),
\end{aligned}
$$

to the problem in Example 1 to the same accuracy in eight iterations. The eigenvalues of the second solution agree with the target eigenvalues to about 14 decimals. As before the error reduction is consistent with second order convergence.

Our empirical evidence suggests that the problem most likely to cause a failure of the method occurs when the number of real and complex eigenvalues 
in one of the iterates differs from the number of real and complex eigenvalues in the target set. In the experience of the authors the method does not recover when this occurs at some stage during the computation. How one might adjust the method to overcome this difficulty remains an open question because of the difficulty of devising a suitable metric for the distance between the current set of eigenvalues and the target set.

We expect, as occurs for the generalized eigenvalue problem case, that the Newton method proposed here can be modified to preserve quadratic convergence (as we expect this method can be shown to have) in the case of equal eigenvalues.

We have presented a new method for pole assignment to a quadratic pencil by using affine sums. The method preserves structure, uses only real arithmetic, and appears to have second order convergence. Further work is necessary to confirm the order of convergence, to establish its behaviour where close or multiple eigenvalues are prescribed and to devise continuation methods for larger distance assignment of the spectrum.

Acknowledgment: The research of Yitshak M. Ram was partially supported by a NSF grant under contract CMS 9978786.

\section{References}

[1] S. Friedland, J. Nocedal, and M. L. Overton. The formulation and analysis of numerical methods for inverse eigenvalue problems. SIAM J. Numer. Anal., 24(3):634-667, 1987. C594

[2] G. M. L. Gladwell. Inverse problems in vibration. Dordecht: Martinus Nijhoff, Nederlands, 1986. C594 
[3] D. Inman. Vibration with control, measurement and stability. Prentice-Hall, Englewood Cilffs, New Jersey, 1989. C593

[4] P. Lancaster and H. Dai. Newton's method for a generalized inverse eigenvalue problem. Numerical Linear Algebra and Applications, 4(1):1-21, 1997. C594

[5] P. Lancaster and J. Maroulas. Inverse eigenvalue problems for damped vibrating system. J. Math. Analysis and Applications, pages 238-261, 1987. C595

[6] P.Lancaster and Qiang Ye. Inverse spectral problems for linear and quadratic matrix pencils. Linear Alg. and Its Appl, 107:293-309, 1988. C595

[7] L. Starek and D. Inman. A symmetric inverse vibration problem with overdamped modes. Journal of Sound Vibration, 181(5):893-903, 1995. C595 